\title{
Theoretical study of solvent influence on the regiospecificity of the reaction of 3-phenyl-s-tetrazine with ketene- $N, N$-aminal
}

\author{
Alexey A. Sinyaev, Maria A. Grishina,* and Vladimir A. Potemkin \\ Chelyabinsk State University, \\ 129, Br. Kashirinih st., Chelyabinsk, 454021, Russia \\ E-mail: maria_grishina@csu.ru
}

\section{Dedicated to Professor Oleg N. Chupakhin on the occasion of his $70^{\text {th }}$ birthday}

(received 31 July 04; accepted 06 Oct 04; published on the web 21 Oct 04)

\begin{abstract}
A theoretical investigation has been performed of the influence of solvent on the regiospecificity of the cycloaddition reaction of 3-phenyl-s-tetrazine with ketene- $N, N$-aminal. In order to reveal the factors determining the ratio of the two possible isomeric products a combined approach including quantum computation, topological analysis, and algorithm MOPS for associate modeling has been suggested. It has been found that the formation of the meta-product of this reaction is thermodynamically controlled. Formation of the ortho- product is preferred in the cases when association of the tetrazine with solvent leads to covering of the reaction centers. The relationships between the ratio of products and characteristics of "tetrazine-enamine", "tetrazine-solvent", "enamine-solvent", "tetrazine-enamine-solvent" complexes have been investigated.
\end{abstract}

Keywords: Regiospecificity, complex modeling, s-tetrazines, cycloaddition reactions, quantum investigations, topological analysis

\section{Introduction}

The theoretical study of reactivity is one of the fundamental problems of organic chemistry. At present, a number of theoretical investigations of processes and structural characteristics of the interacting molecules have been carried out. Usually the consideration of processes includes analysis of the structural characteristics of interacting molecules and the products of a reaction. Although this approach does not explain all of the experimental data, for example the dependence of regiospecificity, equilibrium- or rate- constant on solvent or reagent. Thus, a theoretical study of a process should include the quantitative consideration of the solvent influence, the possibilities of complex formation between reagents, etc. One of the ways to solve 
this problem can be an analysis of "substrate-reagent", "substrate-solvent" complexes, etc. However, complex- modeling is complicated in many cases by the presence of several centers available for complex- formation that leads to a wide variety of potentially possible aggregates. Consequently, an algorithm for complex- modeling should take into account the effects of interaction in the complexes with the estimation of their energy.

\section{Results and Discussion}

In order to solve the problem it has been suggested that one should use a combined approach including quantum computation, topological analysis, and the algorithm MOPS (Modification of algorithm Mech ${ }^{1}$ ). In this algorithm, the search for the lowest energy complex is realized, starting from the geometry of the supposed preliminary complex. The atoms of the molecules in the complex are moved along each of the Hessian modes until the nearest maximum of energy is overcome $^{2}$. Then, using a local-minimum search ${ }^{3-7}$ the received structure is optimized, and if its energy is less than the energy of the initially supposed complex, the geometry of new complex is saved. The process is carried out iteratively for each of the newly defined complexes. The search for complexes is finished when the iteration does not lead to any new complex. Also, in the algorithm MOPS the solvent influence can be taken into account by using a correlation function.

The study of the influence of complex- formation on the process is particularly interesting in the case of cycloaddition reactions of $s$ - tetrazine derivatives. The reason is that $s$ - tetrazine derivatives have many reaction centers and they can interact with structurally related compounds on different pathways ${ }^{8}$. This especially concerns the cases of processes with dienophiles having different substituents at the double (triple) bond, which complicates the reaction pathway's definition. In such cases, analysis of the charge- and orbital- characteristics of the reagents is not sufficient for a conclusion about which of the centers could take part in a reaction. Besides, in many respects the pathway of cycloaddition reactions of tetrazines is dependent on the conditions ${ }^{8,9}$ (for example, on solvent). Therefore, the theoretical analysis of such processes should include the investigation of, "tetrazine-reagent", "tetrazine-solvent", etc., complexes.

In this work, the theoretical study has been provided for the cycloaddition reaction of 3phenyl-s- tetrazine with ketene- $N, N$-aminal giving two isomeric products (Scheme 1 ) the ratio of

which is dependent on the solvent (Table 1) ${ }^{8}$. It has been shown experimentally that the yield of products is not dependent on the polarity and the permittivity of the solvents. Thus, for example, such different solvents as dichloromethane and acetonitrile give the same ratio of isomeric products. In order to explain this fact, a theoretical analysis of this reaction has been carried out. 


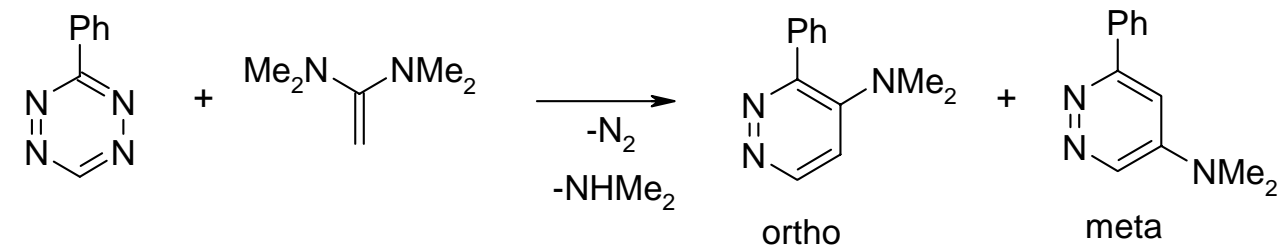

\section{Scheme 1}

Table 1. Solvent influence on the regiospecificity of the reaction presented in Scheme 1

\begin{tabular}{ccc}
\hline Solvent & Yield of ortho- isomer & Yield of meta- isomer \\
\hline$n$-Hexane & 0.78 & 0.22 \\
Toluene & 0.41 & 0.59 \\
Tetrahydrofuran & 0.20 & 0.80 \\
Methyl acetate & 0.14 & 0.86 \\
Acetone & 0.07 & 0.93 \\
Pyridine & 0.04 & 0.96 \\
Dichloromethane & 0.03 & 0.97 \\
Acetonitrile & 0.03 & 0.97 \\
\hline
\end{tabular}

It has been found that the meta- isomer from the process is more stable than the orthoisomer, as can be illustrated by the total energies of the products $(E)$ computed ab initio (HartreeFock 6-31G(d,p)):

$$
E_{\text {ortho }}=-392,367.34 \mathrm{kcal} / \mathrm{mol}, E_{\text {meta }}=-392,376.41 \mathrm{kcal} / \mathrm{mol} \text {. }
$$

Therefore, this product is thermodynamically controlled, while the formation of orthoproduct can be defined by the kinetic factors. In order to reveal the phenomena, the modeling of the, "tetrazine-enamine", "tetrazine-solvent", "enamine-solvent", and "tetrazine-enaminesolvent" complexes provided within algorithm MOPS has been carried out using the MM3 forcefield ${ }^{10-13}$.

Analysis of the "tetrazine-enamine complexes" obtained within MOPS, implicitly taking into account the continuous solvent environment, shows that the most stable complexes are similar. One of them (solvated with acetonitrile) is presented in Figure 1 as an example. The Figure represents that the enamine interacts with the tetrazine by $\pi$-stacking interactions, and provides a reduction of contacts between $\mathrm{C}_{1}$ of the enamine and $\mathrm{C}_{3}$ of the tetrazine (the numbering of the atoms is presented on Scheme 2). Therefore, it allows us to conclude that the most stable complex corresponds to the meta- isomer. 


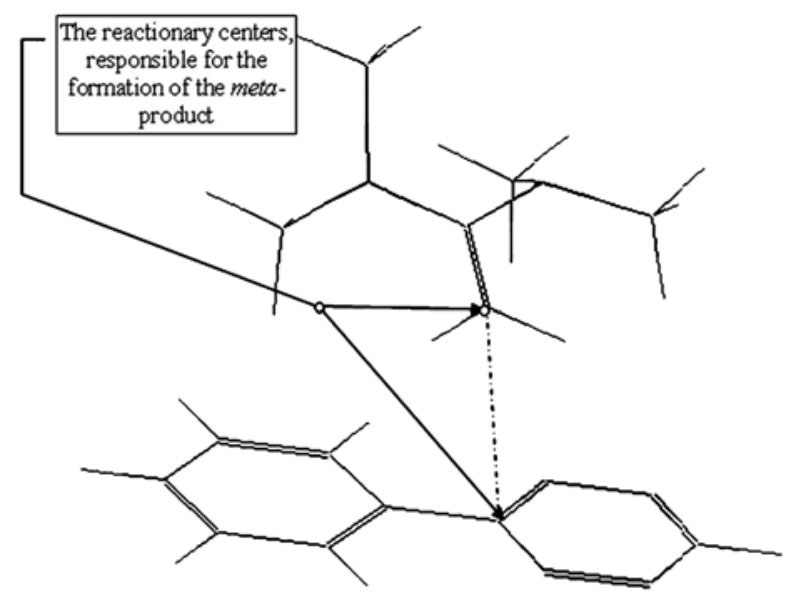

Figure 1. "Tetrazine-enamine" complex obtained within the algorithm MOPS, implicitly taking into account the continuous acetonitrile environment.

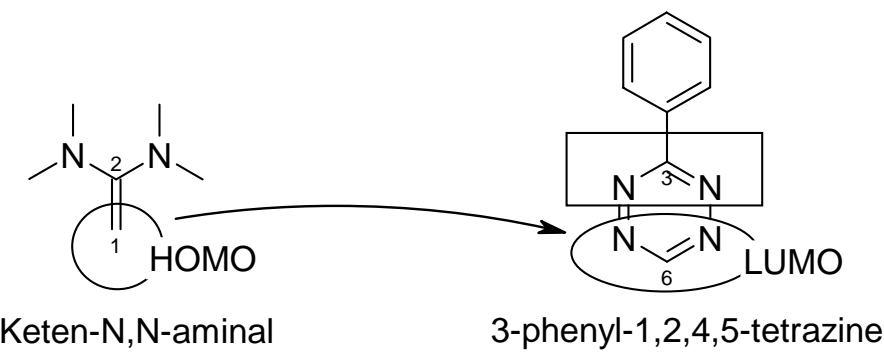

\section{Scheme 2}

Topological analysis of the Laplacian carried out for the "tetrazine-enamine" complexes within the Bader's algorithm ${ }^{14}$ shows four critical points $(3,-1)$ located in the intermolecular space responsible for the interaction of the molecules within each of the complexes (Figure 2). The point 4 has been excluded from further consideration since it is far from the reaction centers and takes less part in the interaction of the molecules than the others. Point 1 is near to the reaction centers: $\mathrm{C}_{1}$ of the enamine and $\mathrm{C}_{3}$ of the tetrazine derivative. Point 2 is under the orthoposition and point 3 is under the meta-position of the tetrazine. Therefore, they should be responsible for the interaction between the nitrogen atoms of the tetrazine ring and the hydrogen atoms of the methyl groups of the enamine. 


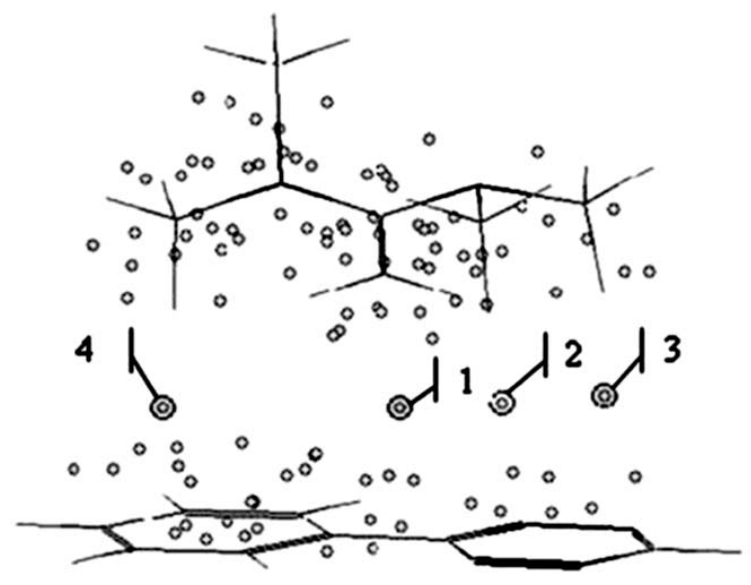

Figure 2. Critical points $(3,-1)$ of Laplacian in the "tetrazine-enamine" complex.

The regression analysis shows the relationship between the yield of the isomers and Laplacian values in the point $1\left(\mathrm{~L}_{1}\right)$ of the complexes:

$$
\begin{aligned}
& \text { Yield }_{\text {meta }}=1-\text { Yield }_{\text {ortho }}=2.20710^{5^{*}} \mathrm{~L}_{1}-2.94710^{3}(\mathrm{r}=0.85) \text { or } \\
& \mathrm{K}_{\mathrm{e}}=\text { Yield }_{\text {ortho }} / \text { Yield }_{\text {meta }}=1.34310^{2}-9.75310^{3^{*}} \mathrm{~L}_{1}(\mathrm{r}=0.82)
\end{aligned}
$$

Therefore, the increasing of the Laplacian value in the critical point leads to an increase of the yield of meta- product owing to the stronger interaction between the $\mathrm{C}_{1}$ - atom of the aminal and the $\mathrm{C}_{3}$ - atom of the tetrazine.

Thus, the most stable "tetrazine-enamine" complex corresponds to the meta-product that also plays an important role in the formation of the meta-isomer. However, simultaneously with the "tetrazine-enamine" complex- formation, the tetrazine and the enamine can associate with solvent forming "tetrazine-solvent" and "enamine-solvent" complexes that can lead to the covering of the reaction centers $\left(\mathrm{C}_{1}\right.$-atom of the enamine and $\mathrm{C}_{3}$-atom of the tetrazine) and change of the orbital distribution in the reagents. It can be the reason for ortho- product formation. In order to estimate the influence of these factors on the process the analysis of the "tetrazine-solvent" and, "enamine-solvent" complexes have been carried out. Ab initio (HartreeFock $\left.6-31 \mathrm{G}(\mathrm{d}, \mathrm{p})^{*}\right)$ computations show that the solvated tetrazine is characterized by lower HOMO (highest occupied molecular orbital) and LUMO (lowest unoccupied molecular orbital) energies than solvated ketene- $N, N$ - aminal in all cases (Table 2).

\footnotetext{
* The computations were performed on the Cluster SKIF of United State of Russia and Belorussia.
} 
Table 2. HOMO and LUMO energies of "tetrazine-solvent" (t-s) and "enamine-solvent" (e-s) complexes

\begin{tabular}{ccccc}
\hline Solvent & $\begin{array}{c}\mathrm{E}_{\text {HOMO }}(\mathrm{t}-\mathrm{s}), \\
\text { a.u. }\end{array}$ & $\begin{array}{c}\mathrm{E}_{\text {LUMO }}(\mathrm{t}-\mathrm{s}), \\
\text { a.u. }\end{array}$ & $\begin{array}{c}\mathrm{E}_{\text {HOMO }}(\mathrm{e}-\mathrm{s}), \\
\text { a.u. }\end{array}$ & $\begin{array}{c}\mathrm{E}_{\text {LUMO }}(\mathrm{e}-\mathrm{s}), \\
\text { a.u. }\end{array}$ \\
\hline Acetone & -0.3330 & 0.0399 & -0.2947 & 0.1565 \\
Acetonitrile & -0.3373 & 0.0294 & -0.2959 & 0.193 \\
Dichloromethane & -0.334 & 0.0391 & -0.3021 & 0.1505 \\
n-Hexane & -0.3341 & 0.0336 & -0.2921 & 0.1978 \\
Methyl acetate & -0.3327 & 0.0375 & -0.2938 & 0.1799 \\
Pyridine & -0.3289 & 0.0422 & -0.2894 & 0.1245 \\
Tetrahydrofuran & -0.3307 & 0.0429 & -0.2947 & 0.1991 \\
Toluene & -0.3095 & 0.0421 & -0.2867 & 0.1423 \\
\hline
\end{tabular}

It can be supposed, therefore, that the important stage of the reaction is electron transfer from the HOMO of the enamine to the LUMO of the tetrazine (as shown on Figure 3), i.e., the tetrazine (diene) plays the role of the electron acceptor, and the enamine (dienophile) the role of electron donor. In this case, it is possible to assume that this cycloaddition reaction is inversely controlled, as has been shown for similar systems earlier ${ }^{[8,15]}$.

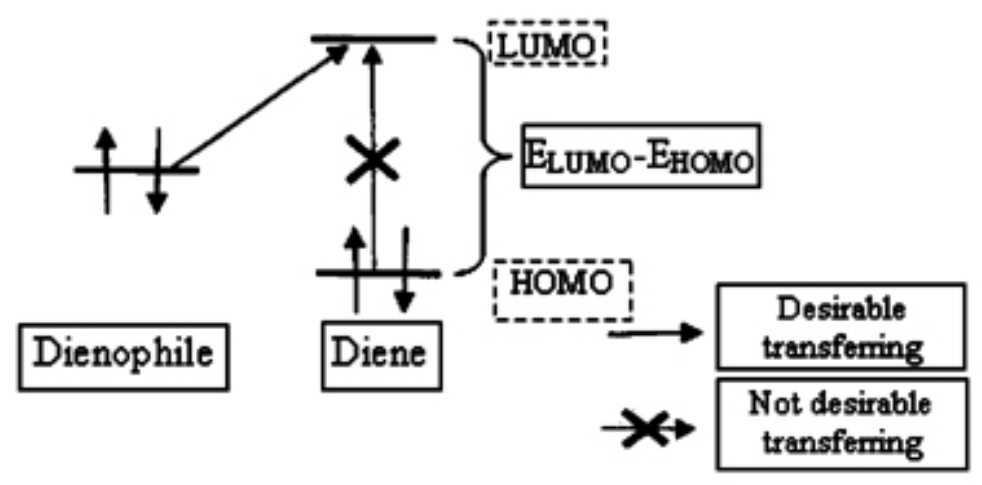

Figure 3. The most probable electron transfer in the tetrazine-enamine system.

Analysis of the orbital characteristics of the, "tetrazine-solvent" and, "enamine-solvent" complexes shows that the $\mathrm{C}_{1}$ - atom of the enamine (selected on Scheme 2 with an ellipse) is characterized by the greatest distribution of the HOMO. In the tetrazine, the LUMO is mainly distributed on $\mathrm{C}_{6}$ and on the nitrogen atoms bonded with it (this region is also selected on Scheme 2 with an ellipse).

The distribution of orbitals allows us to conclude that the most probable electron transfer from HOMO of the solvated enamine to the LUMO of the solvated tetrazine should lead to a 
greater rate of ortho- isomer formation than meta- isomer formation, i.e., the ortho- product can be kinetically controlled.

Thus, the increase of the LUMO distribution ( $\mathrm{p}_{\mathrm{LUMO}, \mathrm{C} 3}$ ) in the region of the $\mathrm{C}_{3}$-atom of the tetrazine ring (this region is marked on Scheme 2 with a rectangle) should reduce the yield of ortho- isomer. In fact, the yield of the ortho- product depends on the orbital distribution in the region of the $\mathrm{C}_{3}$ - atom of the tetrazine (expression 3). Taking into account the difference between LUMO- and HOMO- energies ( $\mathrm{E}_{\mathrm{LUMO}}, \mathrm{E}_{\mathrm{HOMO}}$ ) of the solvated tetrazine, allows us to compute the yield of ortho- product with a correlation coefficient of 0.96 by using the following equation:

$\ln \left(\right.$ Yield $\left._{\text {ortho }}\right)=\ln \left(1-\right.$ Yield $\left._{\text {meta }}\right)=4.483 \times 10^{2}-3.905 \times 10^{2} \mathrm{p}_{\text {LUMO, C3 }}-1.694 \times 10^{2}\left(\mathrm{E}_{\mathrm{LUMO}}-\mathrm{E}_{\mathrm{HOMO}}\right)(3)$

Also, analysis of the "tetrazine-solvent" complexes shows that in the most stable complexes a molecule of solvent is located under the $\mathrm{C}_{3}$ atom of the tetrazine, as it is shown on Figure 4 for the example of the "tetrazine-hexane" complex. This leads to the steric obstacles for the interaction of the solvated tetrazine with the enamine on the pathway to formation of the metaproduct. The stronger interactions in the "tetrazine-solvent" complex provides the lower probability of the reaction with formation of the meta- product.

In fact, topological analysis of electron- density in the, "tetrazine-solvent" complexes fulfilled within Bader's algorithm shows that there are many critical points responsible for the intermolecular interaction of the tetrazine with solvent, as is shown on Figure 4. Increasing the average value of electronic density $(\rho)$ in these points leads to a decreasing yield of the orthoisomer (expression 4). In other words, a solvent competes with the enamine for the complexformation with the tetrazine. Therefore, taking into account the Laplacian value at the critical point $1\left(\mathrm{~L}_{1}\right)$ of the "tetrazine-enamine" complexes it is possible to obtain the ratio of the orthoand the meta- products with correlation coefficient 0.95 .

$\operatorname{lgKe}=\lg \left(\right.$ Yield $_{\text {ortho }} /$ Yield $\left._{\text {meta }}\right)=2.806 \times 10^{2}-2.177 \times 10^{5} \mathrm{~L}_{1}+2.212 \times 10^{2} \rho(\mathrm{r}=0.95)$ 


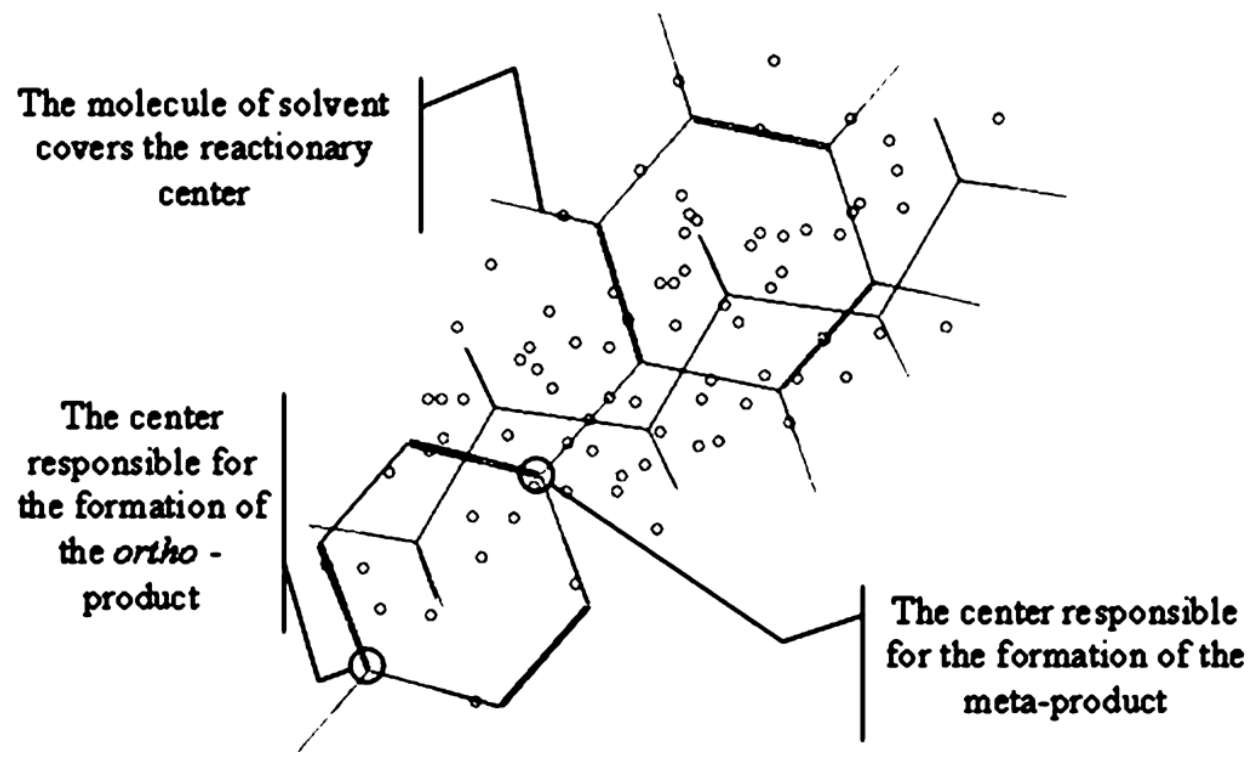

Figure 4. Critical points $(3,-1)$ of the electron- density in the "tetrazine- $n$-hexane" complex.

The competition of the enamine with a solvent for complex- formation with the tetrazine is also confirmed by the modeling of the "tetrazine-enamine-solvent" complexes provided within the algorithm MOPS. It has been shown that, in the case of the solvent coordination near the $\mathrm{C}_{3}$ atom of the tetrazine ring, the steric effects interfere with the attack of the enamine on this reaction center of the tetrazine that leads to the increase of the ortho- product yield. This can be illustrated by the example of the "tetrazine-enamine-hexane" complex represented in Figure 5. The hexane molecule is coordinated closer to the reaction center of the tetrazine (the distance between atom $\mathrm{C}_{3}$ of the tetrazine and the nearest atom of hexane is $3.01 \AA$ ) than the enamine molecule (the distance between the reaction centers is $4.33 \AA$ ). Therefore the yield of the orthoisomer is $78 \%$.

The solvent molecule does not disturb the interaction if it is disposed far from the reaction centers of the tetrazine $\left(\mathrm{C}_{3}\right.$-atom) and the enamine $\left(\mathrm{C}_{1}\right.$-atom), so it defines the primary formation of the meta- product. The "tetrazine-enamine-acetone" complex is presented on Figure 6 as an example of this situation. Here, the reaction center of the enamine molecule is located closer to the $\mathrm{C}_{3}$-atom of the tetrazine (the distance between them is $3.42 \AA$ ) than the nearest atom of the solvent molecule (the distance between the $\mathrm{C}_{3}$-atom of the tetrazine and its nearest atom of the enamine is $4.27 \AA$ ). In fact, the yield of the meta-isomer in acetone solution is $98 \%$. 


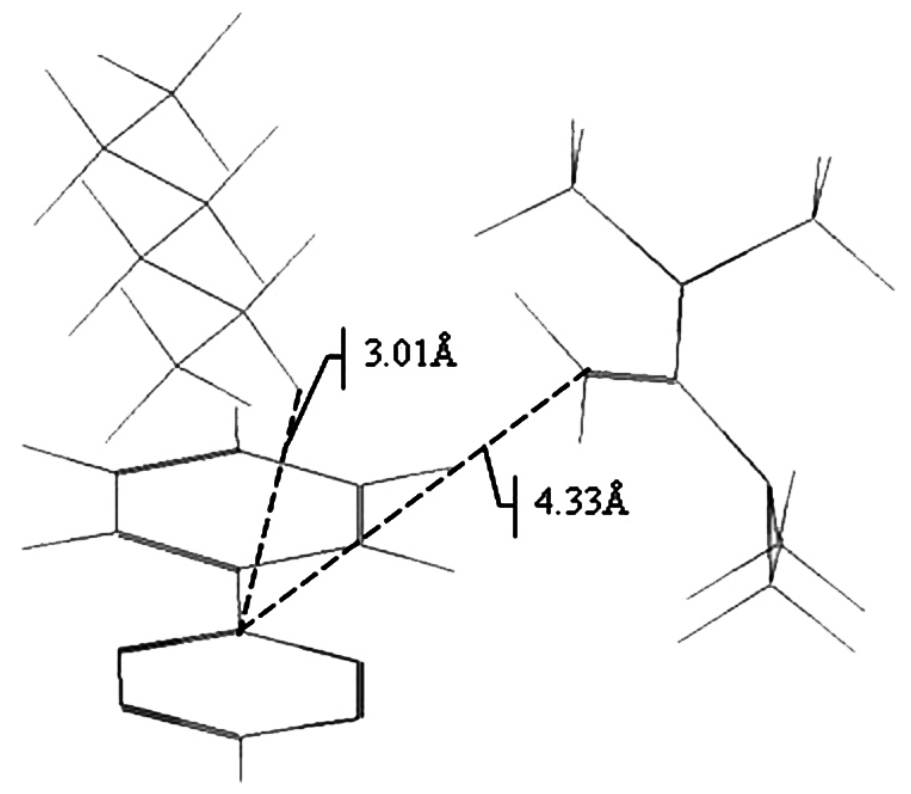

Figure 5. "Tetrazine-enamine- $n$-hexane" complex.

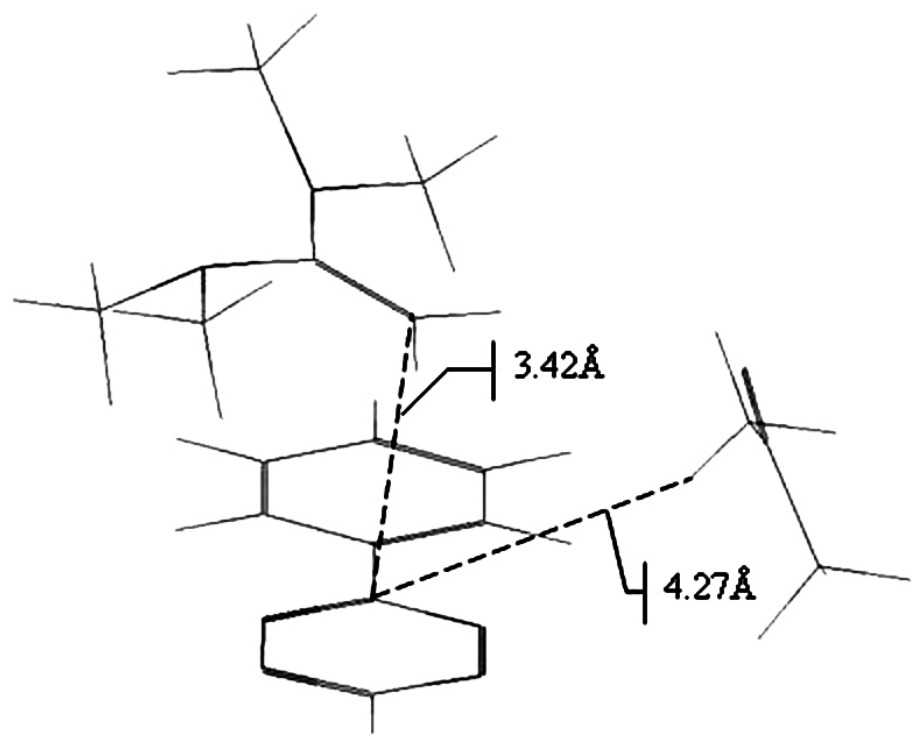

Figure 6. "Tetrazine-enamine-acetone" complex.

Thus, it has been shown that formation of the meta- product of this reaction is defined by the thermodynamic factors: the isomer and the "tetrazine-enamine" complex corresponding to this product are more stable. Although the competition between the solvent and enamine for the complex- formation with tetrazine leads to covering of the reaction centers on the reaction pathway to the meta- isomer. The combined investigation using quantum study, topological analysis, and the MOPS algorithm allows us to explain the regiospecificity of the cycloaddition reaction of 3-Ph- $s$-tetrazine with ketene- $N, N$-aminal depending on the solvent. The relationships 
between the ratio of the isomeric products and the characteristics of the, "substrate-reagent", "reagent-solvent", "substrate-solvent" and "solvent- reagent-substrate" complexes have been found.

\section{Acknowledgments}

This work was possible due to financial support from the supercomputer program SKIF of the United State of Russia and Byelorussia

\section{References}

1. Grishina, M.A.; Bartashevich, E.V.; Potemkin, V.A.; Belik, A.V. J. Struct. Chem. 2002, 6, 1040 .

2. Bartashevich, E.V.; Potemkin, V.A.; Grishina, M.A.; Belik, A.V. J. Struct. Chem. 2002, 6, 1033.

3. Pappu, R.V.; Hart, R.K.; Ponder, J.W. J. Phys. Chem. B 1998, 48, 9725.

4. Griewank, A. O. J. Opt. Theor. Appl. 1981, 34,11.

5. Butler, R. A. R.; Slaminka, E. E. J. Comput. Phys. 1993, 99, 28.

6. Rogers, J. W.; Donnelly, R. A. SIAM J. Optim. 1995, 5, 871.

7. Dembo, R. S.; Steihaug, T. Math. Prog. 1983, 26, 190.

8. Comprehensive Heterocyclic Chemistry II. A review of the literature 1982-1995; Katritzky, A. R.; Rees, C. W.; Scriven, E. F. V., Eds.; Pergamon Press: N. Y., Vol. 6, pp 901-954.

9. Sauer, J. J. Heterocycl. Chem. 1995, 10, 1307.

10. Allinger, N. L.; Li, F.; Yan, L. J. Comput. Chem. 1990, 11, 848.

11. Allinger, N. L.; Li, F.; Yan L., Tai, J. C. J. Comput. Chem. 1990, 11, 868.

12. Lii, J.-H.; Allinger, N. L. J. Comput. Chem. 1998, 19, 1001.

13. Balaban, T. S.; Balaban, A. T.; Foro, S.; Lindner, H.-J. ARKIVOC 2002, (i), 1.

14. Bader, R.F.W. Atoms in Molecules - A Quantum Theory; Clarendon Press: Oxford, 1990; p 256.

15. Rusinov, G. L.; Ishmetova, R. I.; Latosh, N. I.; Potemkin, V. A.; Russ. Chem. Bull. 2000, 2, 354. 